\title{
Integrating Object-Z with Timed Automata
}

\author{
J.S. Dong ${ }^{1} \quad$ R. Duke ${ }^{2}$ P. Hao ${ }^{1}$ \\ ${ }^{1}$ National University of Singapore \\ ${ }^{2}$ University of Queensland, Australia
}

\begin{abstract}
When designing a complex system, Object-Z is a powerful logic-based language for modeling the system state aspects, and Timed Automata is an excellent graph-based notation for capturing timed control behaviour of the system. This paper presents an effective combination of the two techniques with novel composition and communication mechanisms. The combined notation enhances Object- $Z$ with realtime modeling capability and also extends Timed Automata with enhanced structure and state modeling features.
\end{abstract}

Keywords: Specification, Object-Z, Timed Automata

\section{Introduction}

Software system specification is an important activity in software engineering. Formalisms for specifying computer systems have been well researched. Logic-based formalisms, e.g. Z, have been popular in Europe. Graph-based formalisms, e.g. Automata, have been prevalent in North America. The design of complex systems requires techniques for capturing system functionalities and control behaviours. The system functionalities can be best captured in terms of operations and constraints - the ideal application for Z. The system control behaviours can be best captured in terms of visual flows between system functionalities - the ideal application for Automata. Furthermore, complex systems often have intricate system structures and real-time requirements. Object-Z $[11,26]$ is a structured extension to $\mathrm{Z}$ and can be supported by verification tools (e.g. $[24,20,29,34])$. Timed Automata (TA) $[1,36]$ is a realtime extension to Automata and can be effectively verified by model checkers (e.g. [3, 7, 30, 33]).

In our previous work [10], we investigated the projection techniques from the TCOZ [22] (extension to Object-Z) to TA and discussed the notion of timed patterns. In this paper, rather than taking the transformation point of view we propose to develop a novel integrated formal language which combines Object- $Z$ with TA. An effective combination of Object- $Z$ and TA can not only help Object- $Z$ with real-time modeling capability but also help TA with enhanced structure and state modeling features. The result of such a combination can be a powerful unified method for designing complex computer systems. The challenge of achieving an effective combination of Object- $\mathrm{Z}$ and TA is to

- semantically and syntactically link the key language constructs so that the two notations can be used in a cohesive way;

- clearly separate system functionality aspects from time control behaviour patterns so that separate tools can later be applied to check the related system properties;

- consistently unify the composition techniques from both Object-Z (class instantiation) and TA (automaton product) so that subsystem models can be easily and meaningfully composed;

- systematically develop the communication mechanisms so that various concurrent interactions between system components can be precisely captured.

In the remaining sections of this paper we will demonstrate how Object-Z and TA can be effectively combined.

\section{Object-Z and Timed Automata}

In this section, brief introductions to Object- $Z$ and TA are presented together with motivating examples.

\section{Object-Z}

Object- $\mathrm{Z}$ is an extension of the $\mathrm{Z}$ formal specification language to accommodate object orientation. The main reason for this extension is to improve the clarity of large specifications through enhanced structuring.

The essential extension to $\mathrm{Z}$ given by Object- $\mathrm{Z}$ is the class construct which groups the definition of a state schema and the definitions of its associated operations. A class is a template for objects of that class: for each such object, its states are instances of the state schema of the class and its individual state transitions conform to individual operations of 
the class. An object is said to be an instance of a class and to evolve according to the definitions of its class. Syntactically, a class definition is a named box. In this box the constituents of the class are defined and related. The main constituents are: a state schema, an initial state schema and operation schemas. To illustrate Object-Z, consider a simple stock-control system for a store. The store stocks items which each have a fixed use-by date. An item can be added to the store's stock, but only if the use-by date of the added item is today's date or later. Any item can be sold by the store. At the beginning of each day those items whose useby date is less than the current date are removed (i.e. purged) from the store.

To specify this system in Object-Z, first we specify an item as an object of the class Item:

$$
{ }_{\mid \text {useBy }: \mathbb{N}}^{\text {Item }}
$$

Effectively, at this level of abstraction the only important thing about an item is its (fixed) use-by date.

The stock control system is specified by the class Store:

\begin{tabular}{|c|c|}
\hline & _INIT_ \\
\hline $\begin{array}{l}\text { stock }: \mathbb{P} \text { Item } \\
\text { today }: \mathbb{N}\end{array}$ & $\begin{array}{l}\text { stock }=\varnothing \\
\text { today }=0\end{array}$ \\
\hline$a d d$ & _ sell__ _ _ _ \\
\hline $\begin{array}{l}\Delta(\text { stock }) \\
i ?: \text { Item }\end{array}$ & $\Delta($ stock $)$ \\
\hline $\begin{array}{l}i ? \notin \text { stock } \\
i ? . \text { useBy } \geqslant \text { today } \\
\text { stock }=\text { stock } \cup\{i ?\}\end{array}$ & $\begin{array}{l}i ! \in \text { stock } \\
\text { stock }{ }^{\prime}=\text { stock } \backslash\{i !\}\end{array}$ \\
\hline$\left[\begin{array}{l}\text { purge } \\
\Delta(\text { stock, today })\end{array}\right.$ & _purge \\
\hline $\begin{array}{l}\text { today }^{\prime}=\text { today }+1 \\
\text { stock } k^{\prime}=\text { stock } \backslash\{i:\end{array}$ & ock $\mid$ i.useBy $<$ today \\
\hline
\end{tabular}

The semantics of Object- $Z$ can be seen as a state transition system. For example, given a particular Store object state $\sigma=\left\{\left(\right.\right.$ store,$\left\{\right.$ item $_{a}$, item $\left.\left._{b}\right\}\right),($ today, 20$\left.)\right\}$, if operation add is then performed with a new input item item $_{c}$, the new object state would be sigma $=$ $\left\{\left(\right.\right.$ store $\left\{\right.$ item $_{a}$, item $_{b}$, item $\left.\left._{c}\right\}\right),($ today, 20$\left.)\right\}$.

Notice that although there is an attribute today in this class and this attribute is incremented whenever the purge operation takes place, no notion of the progressive passing of time is captured by this specification. Conceptually, we think of the purge operation as taking place once a day, but this is not captured explicitly. Furthermore, in standard Object-Z the operations are assumed to be atomic, so there is no direct way of capturing the idea that an operation may take a specific time to complete.

\section{Timed Automata}

Timed Automata (TA) are finite state machines with clocks. It was introduced as a formal notation to model the behavior of real-time systems. Its definition provides a general way to annotate state-transition graphs with timing constraints using finitely many real-valued clock variables. Another interesting aspect of TA is that there exist model checking methods for temporal logics with quantitative temporal operators which are directly applied to TA. Thus a variety of tools are available for specification and verification of realtime system modeled in TA.

In this paper, we follow the definitions given in [1]. Formally, for a set $X$ of clock variables, the set $\Phi(X)$ of clock constraints $\varphi$ is defined by the following grammar:

$$
\varphi:=x \leq c|c \leq x| x<c|c<x| \varphi_{1} \wedge \varphi_{2}
$$

where $x$ is a clock in $X$ and $\mathrm{c}$ is a constant in $\mathbb{R}$.

A clock interpretation $\nu$ for a set $X$ of clocks assigns a real value to each clock; that is, it is a mapping from $X$ to the set of nonnegative reals. We say that a clock interpretation $\nu$ for $X$ satisfies a clock constraint $\varphi$ over $X$ iff $\varphi$ evaluates to true according to the values given by $\nu$. For $\delta \in \mathbb{R}, \nu+\delta$ denotes the clock interpretation which maps every clock $x$ to the value $\nu(x)+\delta$. For $Y \subseteq X, \nu[Y:=0]$ denotes the clock interpretation for $X$ which assigns 0 to each $x \in Y$, and agrees with $\nu$ over the rest of the clocks.

A timed automaton A is a tuple $\left(S, S_{0}, \Sigma, X, I, E\right)$, where $S$ is a finite set of states/locations; $S_{0}$, a subset of $S$, is a set of initial states; $\Sigma$ is a set of actions/events; $X$ is a finite set of clocks; $I$ is a mapping that labels each location $s$ in $S$ with some clock constraint in $\Phi(X) ; E$, a subset of $S \times S \times \Sigma \times 2^{X} \times \Phi(X)$, is the set of switches. A switch $\left\langle s, s^{\prime}, a, \lambda, \delta\right\rangle$ represents a transition from state $s$ to state $s^{\prime}$ on input symbol $a$. The set $\lambda$ gives the clocks to be reset with this transition, and $\delta$ is a clock constraint over $X$ that specifies when the switch is enabled.

An example of a timed automaton is shown in Figure 1, a gate in a security system. The gate has three states: closed, open and opened (we assume the gate slams shut instantly when directed, so there is no close state). State closed is the initial state, as indicated by there being an action into closed that emanates from no state. Through action open$s$, (i.e. open start) the gate starts the operation of opening which it completes within 2 time units. When the opening operation is completed, the action open-e (i.e. open end) occurs and the gate becomes opened. Through action close the gate closes instantly when exactly 10 time units have elapsed since the gate was opened. 


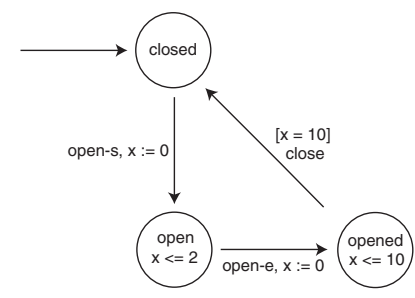

Figure 1. a gate

\section{Combining Object-Z and TA}

In this section, the semantic and syntactic issues on integrating Object- $Z$ and TA are discussed and a combined notation is proposed.

To illustrate how Object-Z and TA can be effectively integrated, consider the simple stock-control system we met in the last Section. What we want is to integrate into this specification a notion of the sequential passing of time.

Suppose time is a positive real number measured in days starting at 0 (so, for example, 1.5 is halfway through the second day). The use-by date associated with an item is a positive integer denoting the day by which the item must be sold or else purged from the store (e.g. a use-by date of 3 means that if the item is not sold on or before day 3 , at the start of day 4 it is purged).

We shall suppose it takes at most $T a$ time units to add an item to the stock, at most $T s$ time units to sell an item in stock, and more than $T p 1$ but less than $T p 2$ time units $(T p 1<T p 2)$ to purge the stock at the beginning of the day, where each of $T a, T s$ and $T p 2$ is much less than 1. Furthermore, the addition of any item to the stock or the selling of any item in stock must be started and completed within the same day. In our model, operations of the store will be disjoint, i.e. time-wise they do not overlap.

The store with this timing information incorporated is specified by adding a Timed Automaton to the class Store to get the class TimedStore as shown. The top part of the class box is the standard Object-Z specification we met in the last section and contains no timing information. The bottom part of the class box contains a declaration of the timing constants and the names of the clocks (in this case there are two clocks, $x$ and $y$ ) as well as the associated automaton. Declaration $x$ : clock means that $x$ plays a dual role: it identifies (i.e. names) a clock and also records the time showing on the clock, i.e. it is a variable that takes positive real number values. In fact, as we shall see, the value of the clock $x$ in this specification always lies between 0 and 1 inclusive and denotes the time that has passed in the current day. The clock $y$ is used to ensure that the operations are completed within the specified time. It is assumed both clocks progress at the same rate, i.e. the passage of time is universally uniform.

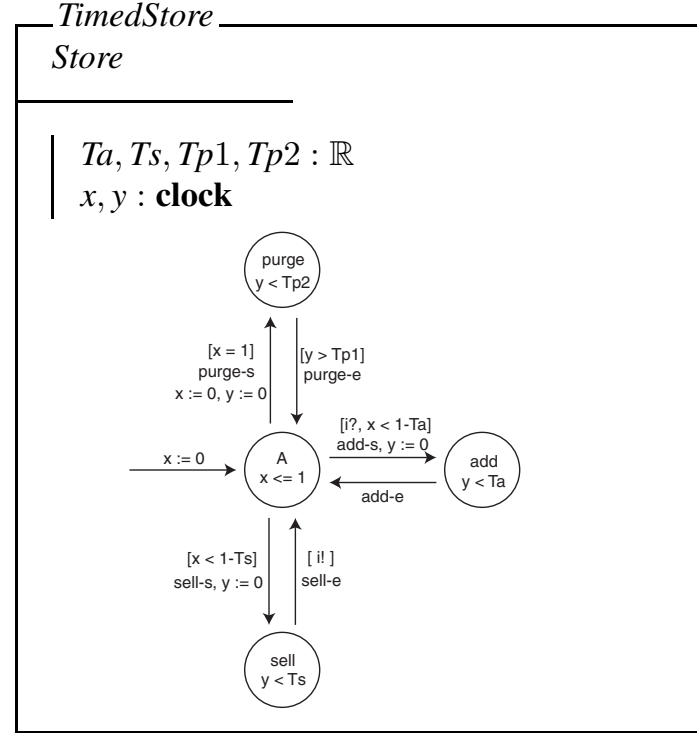

The locations of the automata represent the various situations in which the store can find itself. A location, together with the switches to and from that location, specifies the timing limits (if any) for the corresponding situation. For each of the three operations specified in the Object-Z part there is a similarly-labeled location to capture the situation when the store is undergoing this operation; the store can undergo this operation only when in the corresponding location. The other location, $A$, represents the situation when the store is idle and no operation is being performed.

To illustrate the switches, consider those between locations $A$ and $a d d$. The switch from $A$ to $a d d$ is labeled $a d d-s$ (i.e. add start), while the switch from add to $A$ is labeled add-e (i.e. add end). The expression in square brackets, i.e. $[i ?, x<1-T a]$ in the case of the switch labeled add-s, captures the requirements that must be met if the switch is to take place, i.e. the input item $i$ ? (as defined in the Object- $Z$ operation $a d d$ ) must be supplied, and in addition the time as recorded by the clock $x$ must be less than $1-T a$ (so that the operation, which can take up to $T a$ time units to occur, can be completed within the same day). In addition, the precondition of the Object-Z operation add must hold for the $a d d-s$ switch to occur. As the precondition of an operation must always hold before the switch to the place labeled by that operation's name can occur, this precondition is always implicitly conjoined with any specific additional requirements within the square brackets. When the switch from $A$ to add occurs, the clock $y$ is reset to 0 ; annotating the location add with the condition $y<T a$ ensures that this location is exited within time duration $T a$, as required.

For the operation sell, the supply of the output item $i$ ! is a requirement that must be met for the switch sell-e to occur after the completion of the operation. Compare this with the add operation where the input $i$ ? was required for the switch starting the operation to occur. 
Looking now at the switch purge-s, this switch can occur only when $x$ is 1 . Furthermore, it must occur at this time because of the time restriction placed on location $A$. This ensures that the purge operation occurs precisely once a day (starting at the end of each day and the beginning of the next). When the switch does occur, the clock $x$ is reset to 0 (ensuring that $x$ always lies between 0 and 1 and hence denotes the time that has passed in the current day).

Location $A$ is the automaton's initial location. The understanding is that the initial conditions as specified by the INIT schema must hold when the automaton is started in location $A$, and at the same time the clock $x$ is set to 0 (the initial value of the clock $y$ can be arbitrary and so is not specified). The fact that the 'start' switches associated with each operation emanate from location $A$ and the 'end' switches each return to $A$, ensures that the operations add, sell and purge do not overlap time-wise.

Note that the naming of switches can be systematic, e.g., a switch pointing to an operation state can be labeled with the operation name follow by 's' (for start). If a switch is pointing from an operation state to an idle (control) state, then it can be labeled with the operation name follow by 'e' (for end).

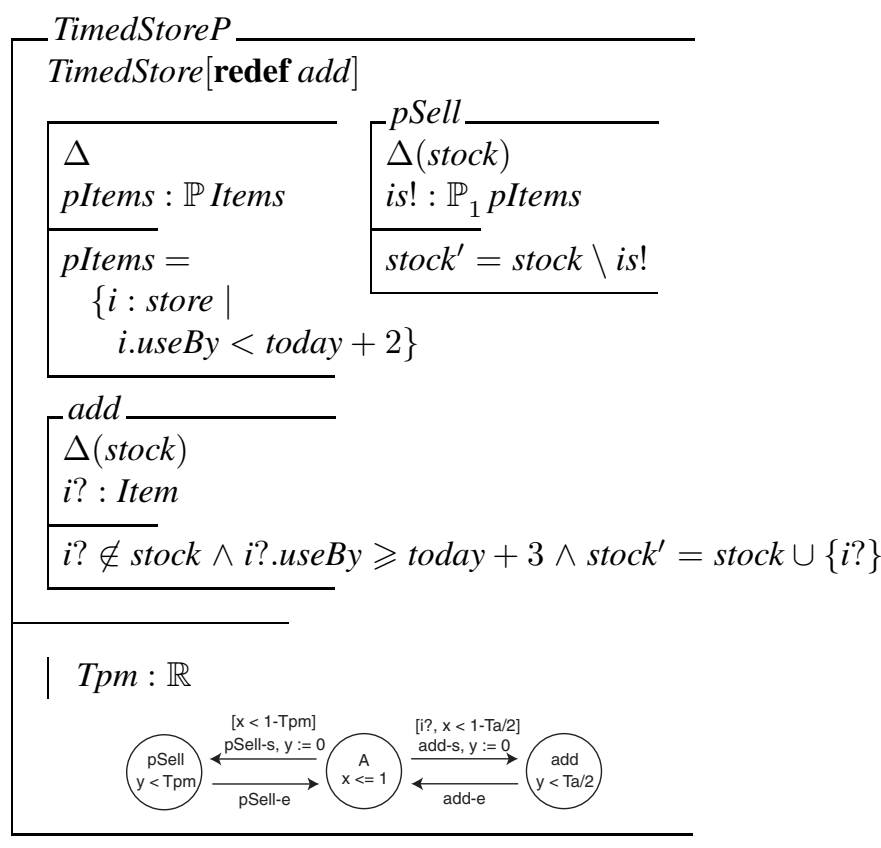

\section{Inheritance}

Inheritance is a mechanism for incremental specification and reuse, whereby new classes may be derived from an existing class. Object- $Z$ inheritance has a similar style as the $\mathrm{Z}$ schema inclusion. We propose that the control behaviour (expressed by the TA) can also be inherited and extended in a simple way. Consider a system TimedStoreP which has the same sell and purge functionalities with TimedStore ex- cept the $a d d$ operation: only items with their expiration date at least 3 days ahead of the current day can be added into the store and the add operation takes less than a half of the $\mathrm{Ta}$ time units to finish. In addition, The system is able to identify the set pItems (promotion items) of items which have only two days left before their expiration. An extra operation $p$ Sell (promotion sell), which takes at most Tpm time units to execute, can sell a subset of these promotion items. The class TimedStoreP can be defined by inheriting the class TimedStore. For the behaviour (automaton) part, the add location refers to the redefined add operation and its local invariant changes to $y<T a / 2$ and enabling condition changes to $x<1-T a / 2$. And a new location $p$ Sell is introduced and is connected (by new switches) with the control (idle) location $A$ from TimedStore. The other locations and their connections remain unchanged as follows: If we expand the inheritance, then TimedStoreP becomes:

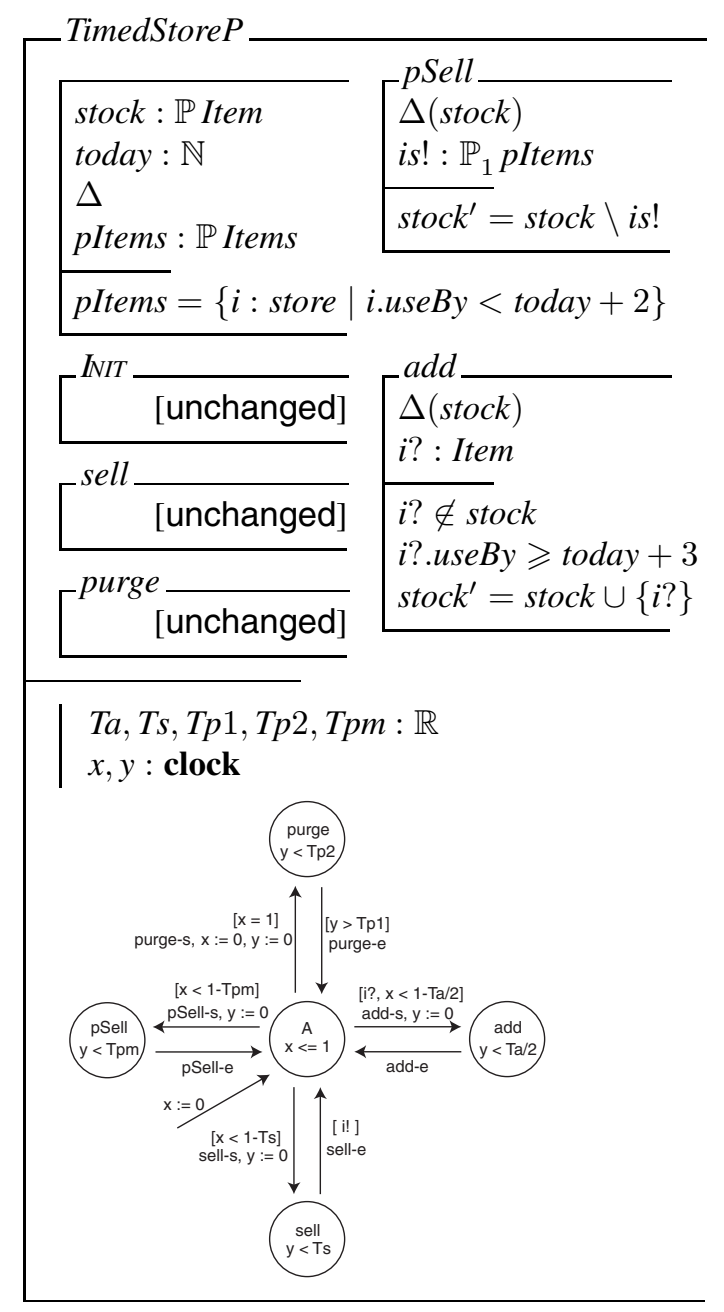

Note that pItems is modelled as a secondary attribute whose value is subject to change with each operation (implicitly it is included in every operation's $\Delta$ list).

For multiple inheritance cases, the rules are that all simi- 
larly named locations (and switches) are merged, with all corresponding invariants and conditions conjoined.

\section{Composition and Communication}

In this section, various composition and communication aspects of the combined language are discussed and synchronized communication links are systematically introduced.

\section{Independent stores}

Consider now a system consisting of two stores operating independently. This system is specified by the class TwoIndStores:

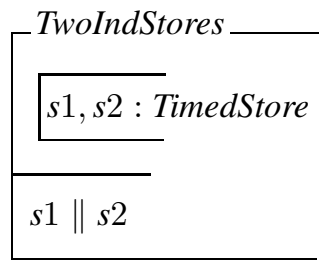

The timed automaton of this class is simply the product [1] of the automata for the two stores $s 1$ and $s 2$. The timed automaton for $s 1$ is just the automaton of the TimedStore class but with the label of each location, the label of each switch, and the names of the clocks distinguished by an ' $s 1$.' prefix, as illustrated in Figure 2. Notice that the input/output variables are not prefixed.

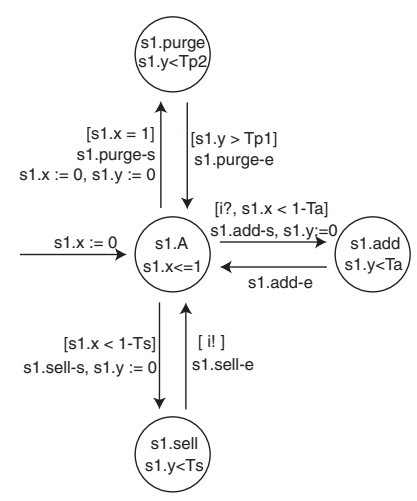

Figure 2. the automaton $s 1$

The timed automaton for $s 2$ is labeled similarly. The $s 1 \| s 2$ notation in the class TwoIndStores denotes the product of the associated automata. The implication here is that the two stores are not only completely independent, but operations in different stores can be executed concurrently. Indeed, when an object of the class TwoIndStores is instantiated, the two store objects start at the same time in their $A$ position with $s 1 . x$ and $s 2 . x$ set to 0 synchronously. As time passes at the same rate for all clocks, both stores will always synchronise on the start of their respective purge operations, namely, at the start of the next day, but apart from that they run completely independently.

The two-stores example can be generalised to a collection of independent stores, as specified by the class CollnIndStores. In this class the expression $\| s:$ stores denotes the timed automata product $\left(s_{1}\left\|s_{2}\right\| \cdots\right)$ where the set stores is $\left\{s_{1}, s_{2}, \cdots\right\}$.

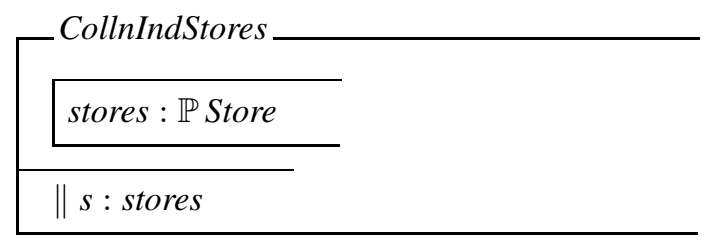

\section{Transferring between stores}

Consider now a system consisting of two stores, where each item sold by the first store is added (i.e. transferred) to the second. Effectively, the first store sells items only to the second store. A specification of this system is given by the class TransStores:

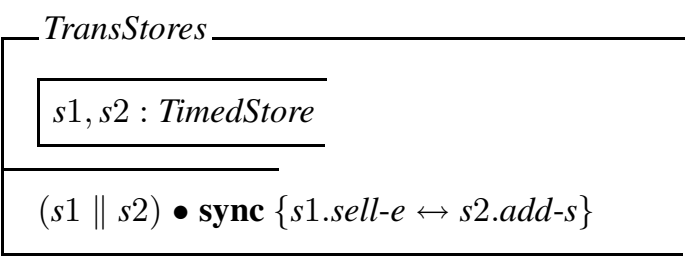

The sync clause indicates that the two switches labeled $s 1 . s e l l-e$ and $s 2 . a d d-s$ are to be treated as if these labels were identical, i.e. the automata must synchronize on these switches. As part of this synchronization, as the output $i$ ! and the input $i$ ? have the same base-name they are identified and hidden (just as is the case for the Object-Z parallel operator, i.e. they specify internal communication rather than communication with the environment). Apart from this synchronization, the product of the two timed automata effectively ensures that the two automata operate independently and concurrently.

Now consider a system like TransStores where again each item sold by the first store is added (i.e. transferred) to the second. However, an item from the environment may also be added to the second store, i.e. not all items added to the second store are necessarily transferring from the first. This system is specified in the class Alt1TransStores:

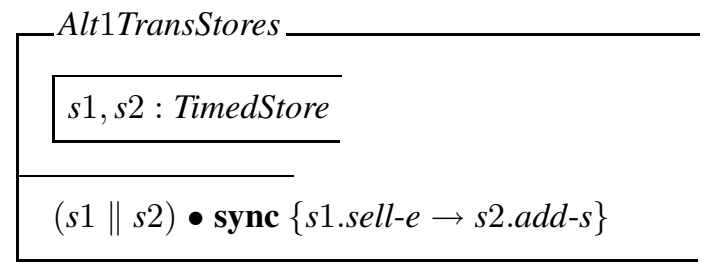


The implication here is that whenever the switch s1.sell$e$ is taken then there must be synchronization with the switch $s 2 . a d d-s$. However, the switch $s 2 . a d d-s$ can occur independent of (i.e. without synchronizing with) the switch s1.sell-e. With this notation, notice that the synchronization sync $\{s 1$. sell-e $\leftrightarrow s 2 . a d d-s\}$ in the TransStores class could have been alternatively (but less elegantly) expressed as sync $\{s 1$.sell-e $\rightarrow s 2$.add-s, s2.add-s $\rightarrow$ s1.sell-e $\}$.

Now consider the situation as before where an item sold by the first store can be transferred to the second, but in addition not only can an item from the environment be directly added to the second store, (i.e. not all items added to the second store are necessarily transferring from the first) but also an item sold by the first store can be passed to the environment (i.e. not all items sold by the first store are necessarily transferred to the second). This system is specified in the class Alt2TransStores:

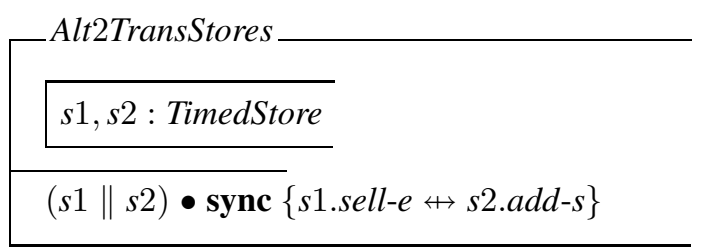

The implication here is that when any of the switches $s 1$.sell-e or $s 2 . a d d-s$ is taken there may or may not (the choice is non-deterministic) be synchronization with the switch $s 2 . a d d-s$ or $s 1$.sell-e respectively.

The examples involving two stores given so far in this section can be generalised to a collection of stores. Consider a system consisting of a collection of stores where an item from the environment can be added to any store, an item sold by any store can be passed back to the environment, and given any two stores in the collection, an item sold by the first store can be added (transferred) to the second. Such a system is specified in the class CollnTransStores:

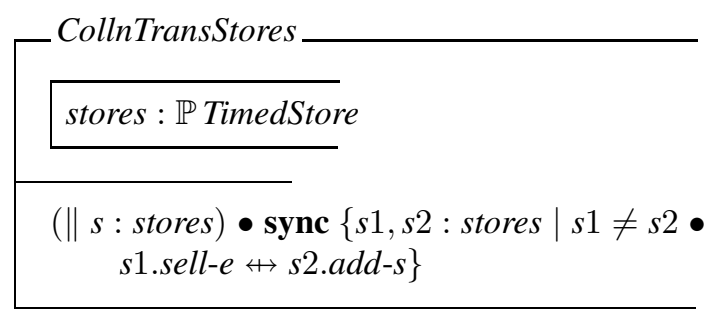

\section{More on synchronization}

To further illustrate synchronization in timed automata, consider the three timed automata $U, V$ and $W$ illustrated in Figure 3 . The timed automaton $(U\|V\| W) \bullet$ sync $\{a \leftrightarrow$ $b\}$ is behaviorally equivalent to the product $U 1\|V 1\| W 1$ of the timed automata $U 1, V 1$ and $W 1$ illustrated in Figure 4 . In this case the switches labeled $a$ and $b$ have been re-named to a common label $d$. As these labels are the same, the product automaton will synchronize on these switches. Consequently, the switch from location $u 1$ to location $u 2$ in $U 1$ is always synchronized with the switch from location $v 1$ to location $v 2$ in $V 1$, and conversely.
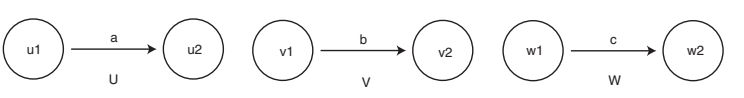

Figure 3. timed automata $U, V$ and $W$

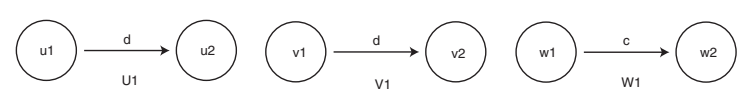

Figure 4. timed automata $U 1, V 1$ and $W 1$

The timed automaton

$$
(U\|V\| W) \bullet \operatorname{sync}\{a \leftrightarrow b, a \leftrightarrow c, b \leftrightarrow c\}
$$

is behaviorally equivalent to the product $U 2\|V 2\| W 2$ of the 3 automata $U 2, V 2$ and $W 2$ illustrated in Figure 5. In this case the switch from location $u 1$ to $u 2$ in $U 2$ must synchronize with either the switch from location $v 1$ to $v 2$ in $V 2$ or from $w 1$ to $w 2$ in $W 2$; the switch from location $v 1$ to $v 2$ in $V 2$ must synchronize with either the switch from location $u 1$ to $u 2$ in $U 2$ or from $w 1$ to $w 2$ in $W 2$; and the switch from location $w 1$ to $w 2$ in $W 2$ must synchronize with either the switch from location $u 1$ to $u 2$ in $U 2$ or from $v 1$ to $v 2$ in $V 2$.

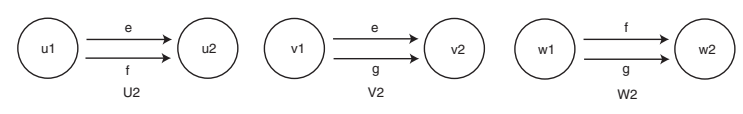

Figure 5. timed automata $U 2, V 2$ and $W 2$

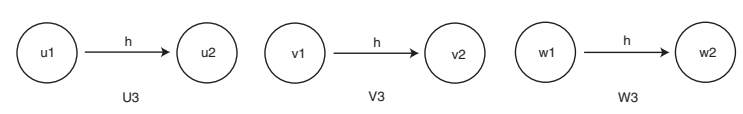

Figure 6. timed automata $U 3, V 3$ and $W 3$

Compare this to the automaton

$$
(U\|V\| W) \bullet \operatorname{sync}\{a \leftrightarrow b \leftrightarrow c\} .
$$

This automaton is behaviorally equivalent to the product $U 3\|V 3\| W 3$ of the three automata $U 3, V 3$ and $W 3$ illustrated in Figure 6. In this case the three switches from location $u 1$ to $u 2$ in $U 3$, from location $v 1$ to $v 2$ in $V 3$ and from location $w 1$ to $w 2$ in $W 3$ must synchronize.

The timed automaton $(U \| V) \bullet$ sync $\{a \rightarrow b\}$ is behaviorally equivalent to the product $U 4 \| V 4$ of the timed automata $U 4$ and $V 4$ illustrated in Figure 7 . In $V 4$ a switch 
labeled $a$ is added to duplicate the switch labeled $b$. As the switch in automaton $U 4$ is also labeled $a$, this ensures that the product automaton will synchronize on these two switches. Consequently, the switch from location $u 1$ to location $u 2$ in $U 4$ is always synchronized with a switch from location $v 1$ to location $v 2$ in $V 4$, but not conversely. The transition from location $v 1$ to location $v 2$ can use the switch labeled $b$ in which case no synchronization takes place.
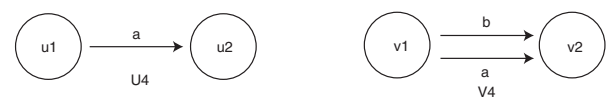

Figure 7. timed automata $U 4$ and $V 4$
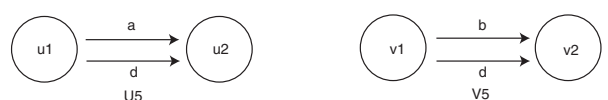

Figure 8. timed automata $U 5$ and $V 5$

The timed automaton $(U \| V) \bullet$ sync $\{a \leftrightarrow b\}$ is behaviorally equivalent to the product $U 5 \| V 5$ of the timed automata $U 5$ and $V 5$ illustrated in Figure 8 . In this case both of the switches labeled $a$ and $b$ are duplicated and a common name, $d$, is assigned to these new switches. This ensures that the product automaton will synchronize on these two switches. Consequently, a transition from location $u 1$ to location $u 2$ in $U 5$ can synchronize with a transition from location $v 1$ to location $v 2$ in $V 5$ if the switch labeled $d$ is used. However, a transition from location $u 1$ to location $u 2$ in $U 5$ could use switch $a$, or a transition from location $v 1$ to location $v 2$ in $V 5$ could use switch $b$; in either case no synchronization takes place.

\section{Semantics}

In this section we present a formal description of the operational behavior of this integrated language. The fundamental semantic links between Object- $Z$ and TA are:

- Object-Z operations are identified as states in Timed Automata.

- Pre/Post-conditions of an Object-Z operation are identified to TA transition conditions.

The key novel idea of integrating the Object- $Z$ semantics and TA semantics is to embed object state updates (of Object-Z) into the action transition semantics of TA. To facilitate the description of dynamic behaviors of a system, we introduce a set of locations $A$, called control locations, to coordinate the location switches from one Object-Z operation to another. Each location of a timed automaton specified in a class must be either a control location or an
Object-Z operation location. A class has an Object-Z part OZDefinition which obeys the conventional definition [26]. OZop represents the set of Object-Z operations defined in the class. The original Object- $Z$ operation operators: parallel composition, nondeterministic choice, sequential composition are replaced by TADefinition, which is a timed automaton:

$\mathbb{S}_{\text {OZTA }}$ is a tuple $\left(S, S_{0}, \Sigma, X, I, E\right)$, where

- $S$ is a union of $A$ and $O p$, in which $A$ is a finite set of control (idle) states and $O p$ is a finite set of operation states correspond to the Object-Z operations

- $S_{0}$, a subset of $S$, is a set of initial locations

- $\Sigma$ is a set of labels

- $X$ is a finite set of clocks

- $I$ is a mapping that labels each location $s$ in $S$ with some clock constraint in $\Phi(X)$

- $E$, a subset of $S \times S \times \Sigma \times 2^{X} \times \Phi(X)$, is the set of switches. A switch $\left\langle s, s^{\prime}, a, r, \varphi\right\rangle$ represents a transition from location $s$ to location $s^{\prime}$ on input symbol $a$. The set $r$ gives the clocks to be reset with this transition, and $\varphi$ is a clock constraint over $X$ that specifies when the switch is enabled.

\section{Operational Semantics}

In this subsection, we present a timed transition system $\mathbb{S}_{O Z T A}$ to represent operational semantic models for this integrated language. Before we start to define the operational semantics, we need some definitions for the validity of Object-Z and TA expressions.

The fact that a state guard $G$ is valid under the semantic function $\sigma:$ Var $\rightarrow$ Value is denoted as $\sigma \vDash G$, which reads that $G$ is valid under the semantic function $\sigma$. The fact that an operation $O p$ is valid under the semantic functions $\sigma_{1}, \sigma_{2}$ is denoted as $\sigma_{1}, \sigma_{2} \vDash O p$. For example, in the context of the Store system,

$$
\begin{aligned}
& \left\{\left(\text { store }\left\{\text { item }_{a}, \text { item }_{b}\right\}\right),(\text { today }, 20)\right\} \\
& \left\{\left(\text { store }\left\{\text { item }_{a}, \text { item }_{b}, \text { item }_{c}\right\}\right),(\text { today }, 20)\right\} \vDash \text { add }\left[i ? \mapsto \text { item }_{c}\right]
\end{aligned}
$$

To keep track of the changes of clock values, we use functions known as clock assignments mapping $X$ to the nonnegative reals $R_{+}$. Let $u, v$ denotes such functions, and use $u \vDash \varphi$ to mean that the clock values denoted by u satisfy the guard $\varphi$. For $d \in R_{+}$, let $u+d$ denote the clock assignment that maps all $x \in X$ to $u(x)+d$, and for $r \subseteq X$, let $[r \mapsto 0] u$ denote the clock assignment that maps all clocks in $r$ to 0 and agree with $\mathrm{u}$ for the other clocks in $X \backslash r$.

To facilitate the description of operational semantics, let

$$
\text { | OP : Location } \rightarrow \text { OZop }
$$


denote the association between TA locations to Object-Z operations.

The operational semantics of this integrated language is an extension of TA transition semantics coupled with object states. The timed state transition system $\mathbb{S}_{O Z T A}$ consists of states which are tuples $\left\langle l, u, \sigma, \sigma_{1}\right\rangle$ and state transitions are defined by the rules:

$$
R_{1}: \frac{\stackrel{a, \varphi, r}{\longrightarrow} l^{\prime} \sigma, \sigma_{1} \vDash O P(l) \sigma_{1}, \sigma_{2} \vDash O P\left(l^{\prime}\right) u \vDash \varphi u^{\prime}=[r \mapsto 0] u u^{\prime} \vDash I\left(l^{\prime}\right) l, l^{\prime} \in O p}{\left\langle l, u, \sigma, \sigma_{1}\right\rangle \stackrel{a}{\longrightarrow}\left\langle l^{\prime}, u^{\prime}, \sigma_{1}, \sigma_{2}\right\rangle}
$$

$R_{1}$ is an action transition from one operation (location) state $l$ to another operation state $l^{\prime}$ where the post object state of $l$ must be the same as the pre object state of $l^{\prime}$, the timing constraints on the transition must be satisfied and the location invariants of $l$ and $l^{\prime}$ must be true.

$$
R_{2}: \frac{\sigma_{1}, \sigma_{2} \vDash O P(l) u \models I(l) u+d \models I(l) d \in R_{+} l \in O p}{\left\langle l, u, \sigma_{1}, \sigma_{2}\right\rangle \stackrel{d}{\longrightarrow}\left\langle l, u+d, \sigma_{1}, \sigma_{2}\right\rangle}
$$

$R_{2}$ is a delay transition in a certain operation state where only time is progressed.

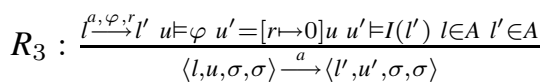

$R_{3}$ is an transition from one control (location) state $l$ to another control state $l^{\prime}$ where object states remain the same.

$$
R_{4}: \frac{u \models I(l) u+d \models I(l) d \in R_{+} l \in A}{\langle l, u, \sigma, \sigma\rangle \stackrel{d}{\longrightarrow}\langle l, u+d, \sigma, \sigma\rangle}
$$

$R_{4}$ is a delay transition in a control state where time is progressed.

$$
R_{5}: \frac{\stackrel{a, \varphi, r}{\longrightarrow} l^{\prime} \sigma_{1}, \sigma_{2} \vDash O P(l) u \vDash \varphi u^{\prime}=[r \mapsto 0] u \quad u^{\prime} \vDash I\left(l^{\prime}\right) \quad l \in O p l^{\prime} \in A}{\left\langle l, u, \sigma_{1}, \sigma_{2}\right\rangle \stackrel{a}{\longrightarrow}\left\langle l^{\prime}, u^{\prime}, \sigma_{2}, \sigma_{2}\right\rangle}
$$

$R_{5}$ is an action transition from one operation (location) state $l$ to a control state $l^{\prime}$ where the post object state of $l$ must be the same as the object state of $l^{\prime}$, the timing constraints on the transition must be satisfied and the location invariants of $l$ and $l^{\prime}$ must be true.

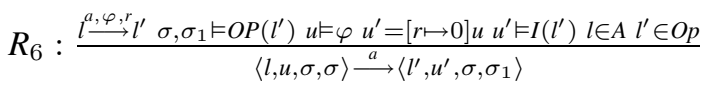

$R_{6}$ is the inverse of $R_{5}$.

These rules define six types of transitions in $\mathbb{S}_{\text {OZTA }}$. These rules are applied to a single timed transition system. A complex system can be described as a product of interacting timed transition systems. The communications between two transition systems are obtained by synchronizing the transition with identical labels.

\section{A Case Study}

As an illustration of how Object-Z and TA can be successfully integrated in practice, we present here a case study of an electronic key system.
A room can be accessed through a sliding door. To open the sliding door, an electronic key is inserted into the door's electronic lock. The identity of the key (as encoded as part of the key) is passed to the lock that then checks to see if the key has permission to access the room. When access permission has been checked the key is ejected from the lock. If the key has access permission the door is opened (or remains open); otherwise the door is closed (or remains closed).

We shall suppose that it takes less than Tsp time units from the time the key is inserted in the door's lock for the key to supply its identity to the lock, less than Tch time units for the lock to check if the key has permission to access the room, less than $T e j$ time units for the key to be ejected from the lock, and less than Top time units for the door to satisfy an 'open' request. Also, if the door has been open Tto time units since the last 'open' request, a time-out occurs and the door is closed. It takes less than $\mathrm{Tcl}$ time units for the door to satisfy a 'close' request.

A key is specified by the class Key:

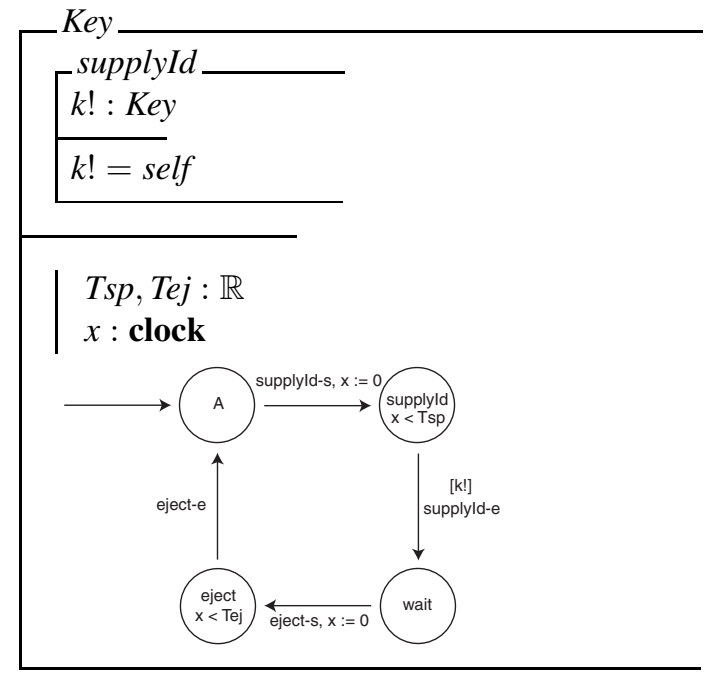

The only operation in the Object- $Z$ section of this class is supplyId specifying the situation in which a key supplies its identity (to the lock). When considering time aspects, however, other situations arise. A key will be in location wait after it has supplied its identity and is waiting to see whether or not access is granted. A key will be in location eject when it is being ejected from the lock once access permission has been decided.

The lock is specified by the class Lock. The attribute keys in this class denotes the set of keys that have permission to access the room. The operations grant and deny capture whether or not any supplied key is in this set, and hence whether or not access to the room is granted or denied. 


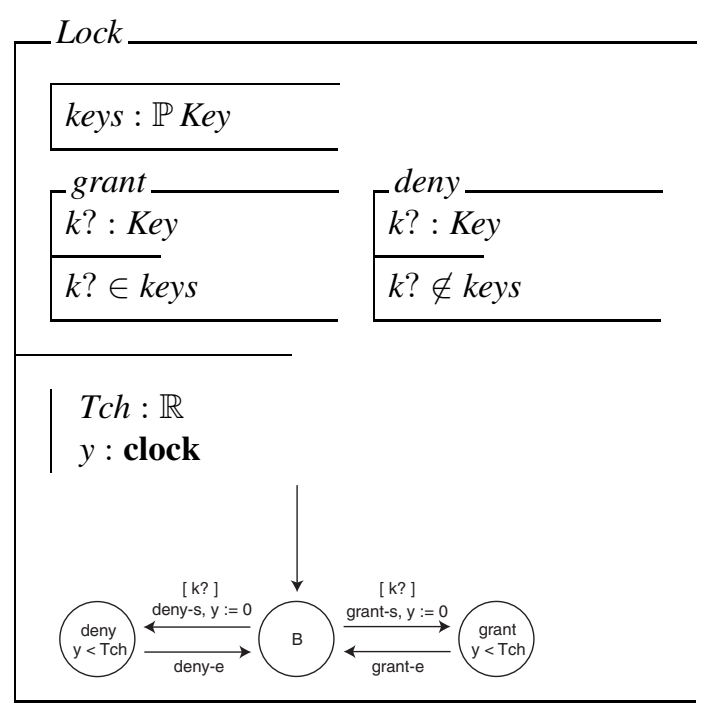

The door is specified by the class Door:

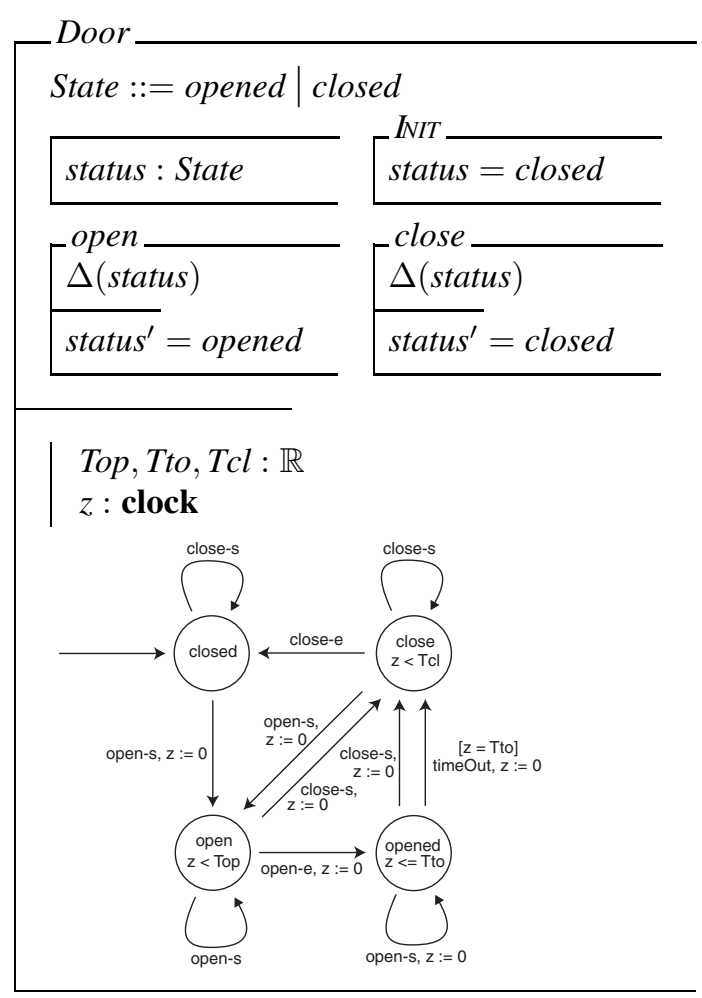

A door can be in any of four situations: closed (location closed) opening (location open where the operation open occurs) opened (location opened) and closing (location close where the operation close occurs). In each situation, the door can receive an instruction to open or close the door. In all cases, when an instruction to open the door is received the switch open-s is taken, while if an instruction to close the door is received the switch close-s is taken. In locations closed or close, if the instruction to open is received the operation open is invoked, while if the instruction to close is received effectively the door continues as if nothing had happened. In location open, if the instruction to open is received effectively the door continues as if nothing had happened, while if the instruction to close is received the operation close is invoked. In location opened, if the instruction to open is received the door remains open but the timing is reset to 0 , while if the instruction to close is received the operation close is invoked.

The complete electronic key system can now be specified by the class KeySystem. In this class, the attribute keys denotes the set of all keys in the system; the set of keys that have permission to access the room will be a subset of keys. The synchronization conditions ensure that the key identity output by a key is passed to the lock and used to determine whether or not that key has permission to access the room, and that once the access permission has been decided the key is ejected and the door requested to open or close, depending on whether access was granted or denied.

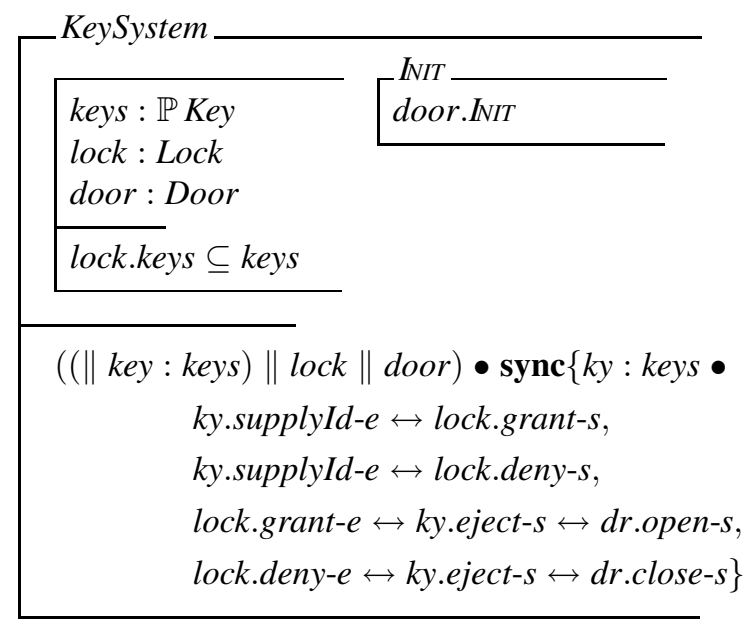

\section{Related Works and Conclusion}

This research can be classified as one of the integrated formal methods (IFM). The IFM research area has been active for a number of years (e.g. [2, 14, 6, 4]) with a particular focus on integrating state based and event based formalisms (e.g. $[13,31,27,32,35])$.

One closely related area to ours is the research work on integration of Object- $Z$ with various timed calculi. For example, Object-Z is combined with Timed CSP [25] in [22, 8], with timed refinement calculus [21, 12] in [28] and with duration calculus [37] in [18]. Indeed, those combinations have made improvements in comparison to some early conservative framework approaches [9, 23].

The technical difference between our approach to the others has been the way to clearly separate functionalities and timed behaviour and the use of graph based Timed Automata instead of the timed calculi to capture the behaviour. 
One clear benefit of our approach is that many existing well developed tools $[3,7,30,33]$ for TA can be used to check the timed behaviour of the design models. In addition to the benefit of bring graphical appeal in capturing the object behaviour of Object-Z classes, our approach also provide a way to structure TA automatons using Object-Z classes so that the scale up problem of TA can be managed. The novel communication mechanism developed in our approach is also more flexible and expressive than CSP channels. For example, arbitrary communication between various objects can be captured at the composite class level with the elegant communication links.

Another related work is the combination of $Z$ with graphical diagrams, i.e. statecharts and petri nets. For example, in [5] a framework is presented to link $\mathrm{Z}$ with statecharts and treats $\mathrm{Z}$ operation schemas as state transition links in statecharts. Similarly, the language OZS [15] blends Object-Z with statecharts and treats Object-Z operations as state transition links. Combinations of $\mathrm{Z}$ and Petri Nets have also been investigated in $[17,16]$. All those approaches suffer a common drawback that the states in the graph have no systematic correspondence in $\mathrm{Z}$ or Object-Z parts. The issues of object composition and timing have not been addressed. Our approach is different: we treat Object- $Z$ operations as states (TA locations) instead of state transitions (TA switches) and furthermore we have a systematic naming convention for all switches. Object composition and real-time issues are the main focus points in our approach. In our future work, we plan to investigate the refinement techniques for this combination of Object- $Z$ and TA. We also plan to develop various tools to support the combination, e.g. to develop an OZTA editor and various linking programs to the Object-Z tools and TA model checkers. For the OZTA editor, we chose to represent the OZTA specification information in an XML format to enable easy linkage with Object-Z and TA tools. Some of our on-going tool development can be accessed here [19].

\section{References}

[1] R. Alur and D. L. Dill. A theory of timed automata. Theoretical Computer Science, 126:183-235, 1994.

[2] K. Araki, A. Galloway, and K. Taguchi, editors. IFM'99: Integrated Formal Methods, York, UK. Springer-Verlag, June 1999.

[3] J. Bengtsson, K. G. Larsen, F. Larsson, and P. Pettersson avd Y. Wang. UPPAAL - a tool suite for automatic verification of real-time systems. In Hybrid Systems III: Verification and Control, pages 232-243. Springer, 1996.

[4] E. Boiten, J. Derrick, and G. Smith, editors. IFM'04: Integrated Formal Methods, Lect. Notes in Comput. Sci. Springer-Verlag, April 2004.

[5] R. Bussow and W. Grieskamp. A Modular Framework for the Integration of Heterogeneous Notations and Tools. In Araki et al. [2], pages 211-230.

[6] M. Butler, L. Petre, and K. Sere, editors. IFM'02: Integrated Formal Methods, Lect. Notes in Comput. Sci. Springer-Verlag, October 2002.

[7] C. Daws, A. Olivero, S. Tripakis, and S. Yovine. The tool KRONOS. In Hybrid Systems III: Verification and Control, pages 208-219. Springer, 1996.

[8] J. Derrick. Timed csp and object-z. In 3nd International Conference of $Z$ and B Users (ZB'03), LNCS. Springer, June 2003.
[9] J. S. Dong, J. Colton, and L. Zucconi. A Formal Object Approach to RealTime Specification. In the 3rd Asia-Pacific Software Engineering Conference (APSEC'96), Seoul, Korea, December 1996. IEEE Press.

[10] J.S. Dong, P. Hao, S.C. Qin, J. Sun, and W. Yi. Timed Patterns: TCOZ to Timed Automata. In Jim Davies and Wolfram Schulte, editors, The 6th International Conference on Formal Engineering Methods (ICFEM'04), Seattle, USA, November 2004.

[11] R. Duke and G. Rose. Formal Object Odiented Specification Using Object-Z. Cornerstones of Computing. Macmillan, March 2000.

[12] C. J. Fidge, I. J. Hayes, A. P. Martin, and A. K. Wabenhorst. A set-theoretic model for real-time specification and reasoning. In Mathematics of Program Construction, 1998.

[13] C. Fischer and H. Wehrheim. Model-Checking CSP-OZ Specifications with FDR. In Araki et al. [2].

[14] W. Grieskamp, T. Santen, and B. Stoddart, editors. IFM'O0: Integrated Formal Methods, Lect. Notes in Comput. Sci. Springer-Verlag, October 2000.

[15] J. P. Gruer, V. Hilaire, A. Koukam, and P. Rovarini. Heterogeneous formal specification based on object-z and startechart: semantics and verification. J. Systems and Software, 2004.

[16] X. He. Pz nets a formal method integrating petri nets with z. Information \& Software Technology, 43(1):1-18, 2001.

[17] M. Heiner and M. Heisel. Modeling safety-critical systems with Z and Petri nets. In International Conference on Computer Safety, Reliability and Security, LNCS, Springer, pages 361-374, 1999.

[18] J. Hoenicke and E.-R. Olderog. Combining Specification Techniques for Processes, Data and Time. In Butler et al. [6].

[19] NUS Software Engineering Lab. OZTA tools. http://ntappn.comp.nus.edu.sg/fm/ozta/introduction.htm.

[20] C. Lüth, E. W. Karlsen, Kolyang, S. Westmeier, and B. Wolff. Hol-Z in the UniForM-workbench - a case study in tool integration for Z. In J. P. Bowen, A. Fett, and M. G. Hinchey, editors, ZUM'98: The Z Formal Specification Notation, volume 1493 of Lect. Notes in Comput. Sci., pages 116-134. SpringerVerlag, 1998.

[21] B. P. Mahony. The Specification and Refinement of Timed Processes. $\mathrm{PhD}$ thesis, University of Queensland, 1991.

[22] B. P. Mahony and J. S. Dong. Blending Object-Z and Timed CSP: An introduction to TCOZ. In K. Futatsugi, R. Kemmerer, and K. Torii, editors, The 20th International Conference on Software Engineering (ICSE'98), pages 95-104, Kyoto, Japan, April 1998. IEEE Press.

[23] K. Periyasamy and V.S. Alagar. Adding Real-Time Filters to Object-Oriented Specification of Time Critical Systems. In the 1998 IEEE Workshop on Industrial-strength Formal specification Techniques, Boca Raton, Florida, USA, October 1998. IEEE Press.

[24] M. Saaltink. The Z/EVES system. In J. P. Bowen, M. G. Hinchey, and D. Till, editors, ZUM'97: Z Formal Specification Notation, volume 1212 of Lecture Notes in Computer Science, pages 72-85. Springer-Verlag, 1997.

[25] S. Schneider, J. Davies, D. M. Jackson, G. M. Reed, J. N. Reed, and A. W. Roscoe. Timed CSP: Theory and practice. In J. W. de Bakker, C. Huizing, W. P. de Roever, and G. Rozenberg, editors, Real-Time: Theory in Practice, volume 600 of Lect. Notes in Comput. Sci., pages 640-675. Springer-Verlag, 1992.

[26] G. Smith. The Object-Z Specification Language. Advances in Formal Methods. Kluwer Academic Publishers, 2000.

[27] G. Smith and J. Derrick. Specification, Refinement and Verification of Current Systems - An Integration of Object-Z and CSP. Formal Methods in System Design, 18:249-284, 2001.

[28] G. Smith and I. Hayes. Towards Real-Time Object-Z . In Araki et al. [2].

[29] G. Smith, F. Kammuller, and T. Santen. Encoding object-z in isabelle/hol. In 2nd International Conference of $Z$ and $B$ Users (ZB'02), LNCS. Springer, 2002.

[30] M. Sorea. TEMPO: A model-checker for event-recording automata. In Proceedings of Workshop on Real-time Tools, August 2001.

[31] C. Suhl. RT-Z: An integration of $Z$ and timed CSP. In Araki et al. [2].

[32] K. Taguchi and K. Araki. The State-Based CCS Semantics for Concurrent $\mathrm{Z}$ Specification. In M. Hinchey and S. Liu, editors, the IEEE International Conference on Formal Engineering Methods (ICFEM'97), pages 283-292, Hiroshima, Japan, November 1997. IEEE Press.

[33] S. Tasiran, R. Alur, R. P. Kurshan, and R. K. Brayton. Verifying abstractions of timed systems. In Proceedings of the 7th Conference on Concurrency Theory, volume 1119 of $L N C S$, pages 546-562. Springer, 1996.

[34] K. Winter and R. Duke. Model Checking Object-Z Using ASM. In Butler et al. [6], pages 165-184.

[35] J. Woodcock and A. Cavalcanti. The Semantics of Circus. In 2nd International Conference on $Z$ and B, volume 2272 of Lect. Notes in Comput. Sci., pages 184-203. Springer-Verlag, 2002.

[36] X.Nicollin, J.Sifakis, and S.Yovine. Compiling Real-time Specifications into Extended Autoamta. In IEEE TSE Special Issue on Real-Time Systems, volume 18(9), pages 794-804, 1999.

[37] C. Zhou, C. A. R. Hoare, and A. P. Ravn. A calculus of durations. Information Processing Letters, 40:269-276, 1991. 